Cent ral and tensor components of three- nucl eon for ces i n l ow energy prot on- deut er on scat tering

\begin{tabular}{|l|l|}
\hline 著者 & I shi kawa Soui chi , Tani fuj i M, I ser i Y. \\
\hline 出版者 & The Aner i can Physi cal Soci et y \\
\hline $\begin{array}{l}\text { j our nal or } \\
\text { publ i cat i on ti t l e }\end{array}$ & Physi cal Revi ew C \\
\hline number & 6 \\
\hline page r ange & $061001-1$ - 061001- 5 \\
\hline year & 2003-06 \\
\hline URL & ht t p: //hdl . handl e. net /10114/1244 \\
\hline
\end{tabular}




\title{
Central and tensor components of three-nucleon forces in low-energy proton-deuteron scattering
}

\author{
S. Ishikawa* and M. Tanifuji \\ Department of Physics, Science Research Center, Hosei University, 2-17-1 Fujimi, Chiyoda, Tokyo 102-8160, Japan \\ Y. Iseri \\ Department of Physics, Chiba-Keizai College, 4-3-30 Todoroki-cho, Inage, Chiba 263-0021, Japan
}

(Received 2 March 2003; published 13 June 2003)

\begin{abstract}
Contributions of three-nucleon forces (3NF) to proton-deuteron scattering observables at energies below the deuteron breakup threshold are studied by solving the Faddeev equation that includes the Coulomb interaction. At $E_{p}=3.0 \mathrm{MeV}$, we find that the central part of a two-pion exchange $3 \mathrm{NF}$ removes the discrepancy between measured cross sections and the calculated ones by two-nucleon forces, and improves the agreement with $T_{22}$ experimental data. However, the tensor part of the $3 \mathrm{NF}$ fails in reproducing data of the analyzing power $T_{21}$ by giving worse agreement between the measured and the calculated. Detailed examinations of scattering amplitudes suggest that a $P$-wave contribution in spin-quartet tensor amplitudes has unsuitable sign for reproducing the $T_{21}$ data.
\end{abstract}

DOI: 10.1103/PhysRevC.67.061001

PACS number(s): 25.10.+s, 21.30.-x, 24.70.+s

Contributions of three-nucleon forces (3NF) have been studied extensively for proton-deuteron $(p d)$ scattering observables, since the interactions are successful [1] in solving the problem of three-nucleon $(3 N)$ underbinding for realistic two-nucleon forces (2NF). However, the Faddeev calculation, which is one of the practical methods to treat $3 \mathrm{~N}$ systems, has conventionally neglected the Coulomb interaction because of mathematical difficulties, while the interaction is essentially important in the low-energy $p d$ scattering. Recently the problem has been solved by an Faddeev integral equation approach at energies below the deuteron breakup threshold [2], where the phase-shift parameters by the calculation agree to those by an Faddeev differential equation approach $[3,4]$ as well as the Kohn variational method $[5,4]$ with very good accuracy. This allows us to investigate the contribution of $3 \mathrm{NF}$ in the low-energy $p d$ scattering by the Faddeev calculation including the Coulomb interaction. In this Rapid Communication, we will report on the main result of such calculations, where one will find remarkable effects of the $3 \mathrm{NF}$ with promising success of the central part but failure of the tensor one.

In the calculation, we adopt the Argonne $V_{18}$ model (AV18) [6] for the 2NF and the Brazil model (BR) [7] for the two-pion exchange 3NF. Further, we introduce two kinds of 3NF: a spin-independent Gaussian (GS) 3NF [2],

$$
V_{\mathrm{GS}-3 \mathrm{NF}}=V_{0}^{G} \sum_{i \neq j \neq k} \exp \left\{-\left(\frac{r_{i j}}{r_{G}}\right)^{2}-\left(\frac{r_{k i}}{r_{G}}\right)^{2}\right\},
$$

with $V_{0}^{G}=-40 \mathrm{MeV}$ and $r_{G}=1.0 \mathrm{fm}$; a spin-orbit (SO) $3 \mathrm{NF}[8]$,

$$
V_{\mathrm{SO}-3 \mathrm{NF}}=\frac{1}{2} W_{0} \exp \{-\alpha \rho\} \sum_{i>j}\left[\boldsymbol{l}_{i j} \cdot\left(\boldsymbol{\sigma}_{i}+\boldsymbol{\sigma}_{j}\right)\right] \hat{P}_{11},
$$

\footnotetext{
*Electronic address: ishikawa@i.hosei.ac.jp
}

where $\quad \rho^{2}=\frac{2}{3}\left(r_{12}^{2}+r_{23}^{2}+r_{31}^{2}\right), \quad \alpha=1.5 \mathrm{fm}^{-1}, \quad W_{0}=-20$ $\mathrm{MeV}$, and $\hat{P}_{11}$ is the projection operator to the spin- and isospin-triplet state of the $(i, j)$ pair. Calculated ${ }^{3} \mathrm{He}$ binding energies are $7.79 \mathrm{MeV}$ for the $\mathrm{BR}-3 \mathrm{NF}$ in addition to the AV18 (AV18-BR), $7.74 \mathrm{MeV}$ for the GS-3NF (AV18-GS), and $7.74 \mathrm{MeV}$ for the BR- and SO-3NF (AV18-BR-SO), which are compared with the empirical value of $7.72 \mathrm{MeV}$. The GS-3NF simulates the central part of the BR-3NF [9], and then the difference between both calculations describes the contribution of the tensor part of the BR-3NF. The SO$3 \mathrm{NF}$ is adopted as a simulation of the spin-vector-type $3 \mathrm{NF}$ that reproduces empirical vector analyzing powers, but the origin is yet unknown at present.

The comparison between the calculated quantities and the measured ones [10-12] is shown in Figs. 1-3 for $E_{p}$ $=0.65,2.5$, and $3.0 \mathrm{MeV}$, respectively, where the differential cross section $d \sigma / d \Omega$, the vector analyzing power of the proton $A_{y}$, that of the deuteron $i T_{11}$, and the tensor analyzing powers $T_{20}, T_{21}$, and $T_{22}$ are displayed. Although overall agreements are obtained between the calculations and the experimental data at the three incident energies, the BR-3NF seems to deteriorate the agreement to the $T_{21}$ data of middle angles at $E_{p}=2.5$ and $3.0 \mathrm{MeV}$. This effect can be confirmed by $\chi^{2} /$ data in Table I, where we include similar analyses of the data [13] at $E_{p}=1.0 \mathrm{MeV}$ to see energy dependence of $\chi^{2}$ in detail.

To demonstrate characteristic features of such BR-3NF contributions, we will show, in Fig. $4, d \sigma / d \Omega, T_{20}, T_{21}$, and $T_{22}$ typically at $E_{p}=3.0 \mathrm{MeV}$, divided by the theoretical values obtained by the AV18 calculations. In the figure, the solid horizontal lines (the 2NF lines) describe the AV18 calculations, and the deviations of the theoretical curves from the $2 \mathrm{NF}$ lines describe the $3 \mathrm{NF}$ contributions. The experimental points of $d \sigma / d \Omega$ deviate from the $2 \mathrm{NF}$ line with characteristic angular distribution, and the deviation is well reproduced by the calculations including the $3 \mathrm{NF}$ effects. This $3 \mathrm{NF}$ effect is attributed to the central part of the BR$3 \mathrm{NF}$, because the calculations by the AV18-BR and those by 

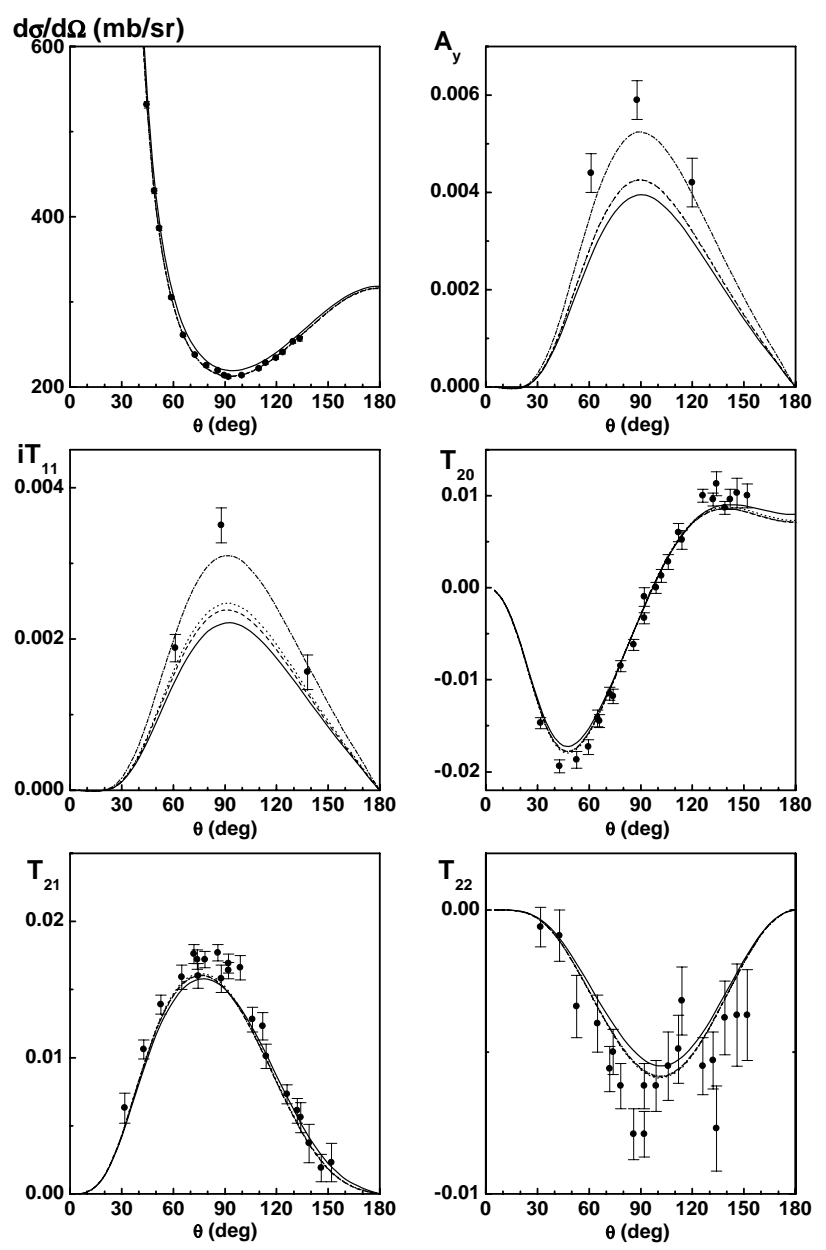

FIG. 1. Differential cross sections $d \sigma / d \Omega$, vector analyzing powers $A_{y}$ and $i T_{11}$, and tensor analyzing powers $T_{20}, T_{21}$, and $T_{22}$ of the $p d$ scattering at $E_{p}=0.65 \mathrm{MeV}$. The data are taken from Ref. [10]. The solid, dashed, dotted, and dash-dotted lines are the calculations by the AV18, the AV18-BR, the AV18-GS, and the AV18-BR-SO, respectively.

the AV18-GS give similar results, and the additional SO-3NF produces very small contributions. As was discussed in Ref. [9], this $3 \mathrm{NF}$ contribution is produced through the spindoublet scalar amplitude and is related to the $3 \mathrm{NF}$ contribution in the $3 N$ binding energy [14].

Contrary to such success, the BR-3NF fails in reproducing the experimental data of $T_{21}$. Figure 4 shows that $T_{21}$ calculated by the AV18-BR at middle angles is located in the opposite side of the measured values with respect to the $2 \mathrm{NF}$ line, indicating the opposite sign of the $3 \mathrm{NF}$ contribution to be desirable for reproducing the data. This failure is dominantly due to the tensor part of the BR-3NF, since the contributions of the GS-3NF and the SO-3NF are small. Similar discrepancy is also observed in $T_{20}$, but the role of the tensor part is not clear. The agreement with the $T_{22}$ data is improved, though is not sufficient, by taking account of the $3 \mathrm{NF}$, where the dominant contribution of the $3 \mathrm{NF}$ comes from the central part of the BR-3NF since three calculations by the AV18-BR, the AV18-GS, and the AV18-BR-SO calculations give similar results.
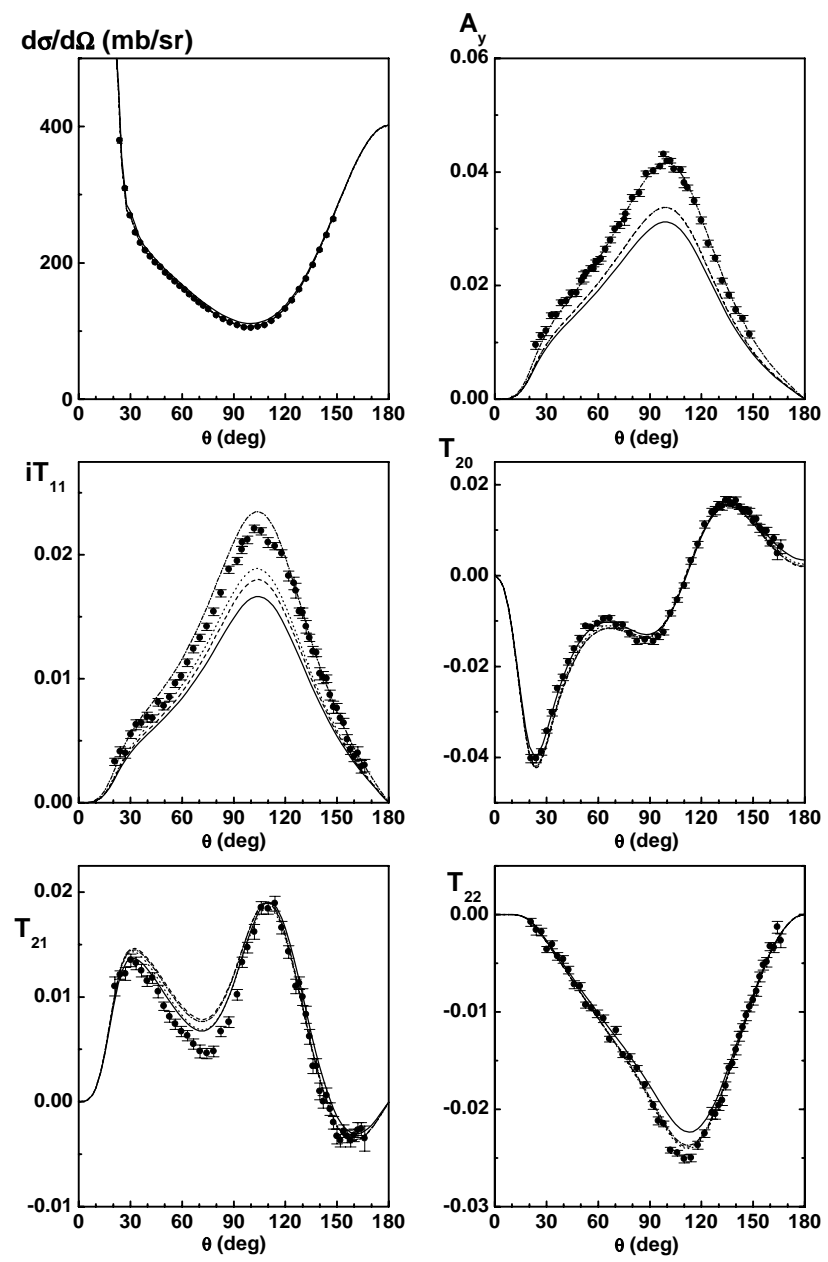

FIG. 2. Same as Fig. 1, but at $E_{p}=2.5 \mathrm{MeV}$. The data are taken from Refs. [11,12].

Next we will examine the $3 \mathrm{NF}$ contributions in more detail for $T_{21}$ and $T_{22}$. To understand the roles of the central interaction and the tensor one of the 3NF individually, we will decompose the scattering amplitude according to the tensor property in the spin space by expanding the $T$ matrix $\boldsymbol{M}$ into the spin-space tensors $\boldsymbol{S}_{\kappa}^{(K)}$ [9],

$$
\boldsymbol{M}=\sum_{K \kappa}(-)^{\kappa} \boldsymbol{S}_{-\kappa}^{(K)} \boldsymbol{R}_{\kappa}^{(K)},
$$

where $\boldsymbol{R}_{\kappa}^{(K)}$ is the coordinate-space tensor and $K(\kappa)$ is the rank ( $z$ component) of the tensor. Then the scattering amplitude is given by Ref. [15] as

$$
\begin{aligned}
& \left\langle\nu_{p}^{\prime} \nu_{d}^{\prime} ; \boldsymbol{k}_{f}|\boldsymbol{M}| \nu_{p} \nu_{d} ; \boldsymbol{k}_{i}\right\rangle \\
& =\sum_{s_{i} s_{f}}\left(s_{p} s_{d} \nu_{p} \nu_{d} \mid s_{i} \nu_{i}\right)\left(s_{p} s_{d} \nu_{p}^{\prime} \nu_{d}^{\prime} \mid s_{f} \nu_{f}\right)(-)^{s_{f}-\nu_{f}} \\
& \quad \times \sum_{K}\left(s_{i} s_{f} \nu_{i}-\nu_{f} \mid K \kappa\right) M_{\kappa}^{(K)}\left(s_{i} s_{f}\right)
\end{aligned}
$$

and 

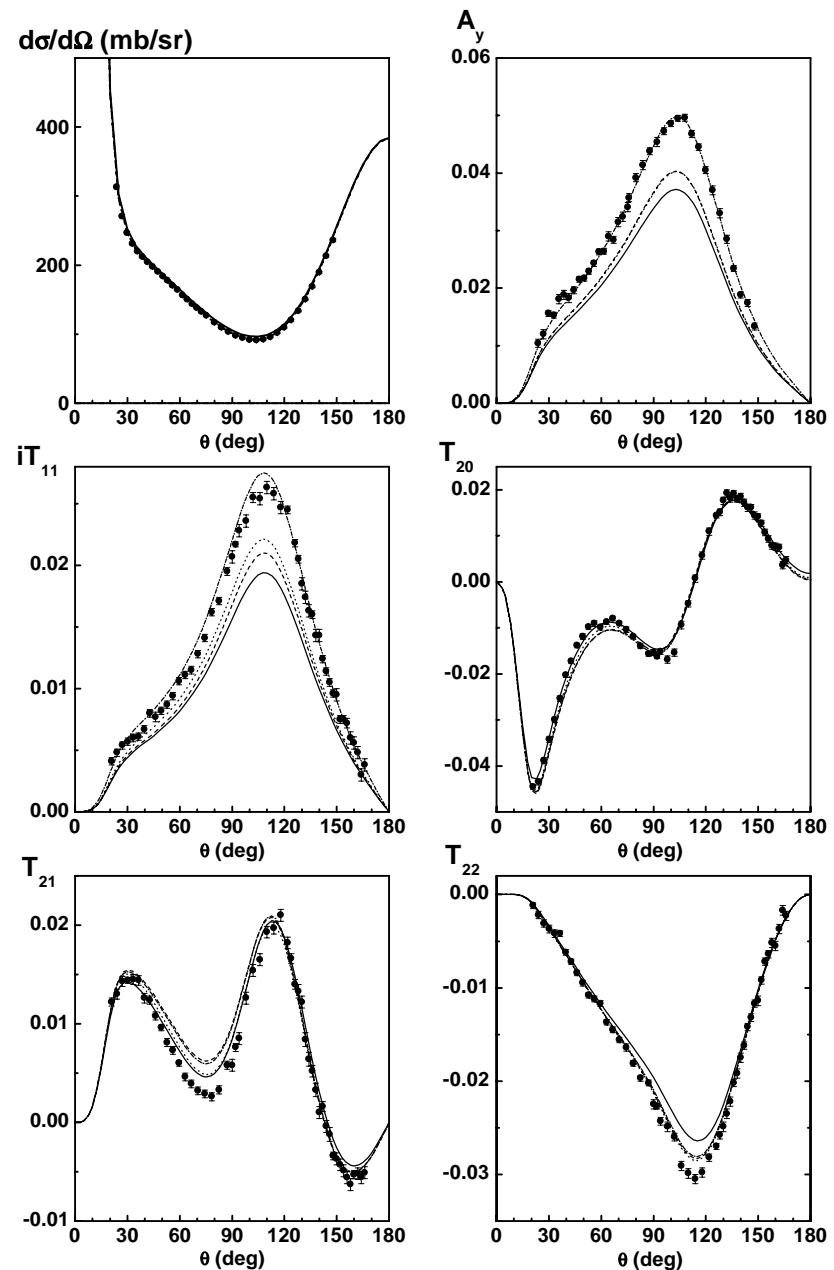

FIG. 3. Same as Fig. 1, but at $E_{p}=3.0 \mathrm{MeV}$. The data are taken from Refs. [11,12].

$$
\begin{aligned}
M_{\kappa}^{(K)}\left(s_{i} s_{f}\right)= & \sum_{\ell_{i}=\bar{K}-K}^{K}\left[C_{\ell_{i}}\left(\hat{k}_{i}\right)\right. \\
& \left.\otimes C_{\ell_{f}=\bar{K}-\ell_{i}}\left(\hat{k}_{f}\right)\right]_{\kappa}^{(K)} F^{(K)}\left(s_{i} s_{f} \ell_{i}\right),
\end{aligned}
$$

where $\bar{K}=K(K+1)$ for $K=$ even (odd), and $C_{\ell m}(\hat{k})$ is related to $Y_{\ell m}(\hat{k})$ as usual [9]. The quantity $s_{i}\left(s_{f}\right)$ denotes the channel spin in the initial (final) state, which is $\frac{1}{2}$ (the spindoublet states) or $\frac{3}{2}$ (the spin-quartet states). The function $F^{(K)}\left(s_{i} s_{f} \ell_{i}\right)$ (the invariant amplitude) is a function of the scattering angle $\theta$, and is designated by the tensor rank $K$. Thus the amplitude describes the scattering by interactions classified by $K$, i.e., $F^{(0)}\left(s_{i} s_{f} \ell_{i}\right)\left[F^{(2)}\left(s_{i} s_{f} \ell_{i}\right)\right]$ describes the scattering by the central (tensor) interactions. Because of the time reversal theorem,

$$
F^{(K)}\left(s_{f} s_{i} l_{f}\right)=(-)^{s_{i}-s_{f}} F^{(K)}\left(s_{i} s_{f} l_{i}\right),
$$

only five amplitudes of nine tensor ones are independent [9].

In the low-energy scattering, the scalar amplitudes dominate other amplitudes as shown in Fig. 5, where the magnitudes of the two scalar and five independent tensor amplitudes at $\theta=90^{\circ}$ are displayed as functions of $E_{p}$. Thus we will apply to the tensor analyzing powers $T_{2 \kappa}(\kappa=0,1,2)$ an approximation in which terms not including the scalar amplitudes are neglected, and get the following expressions:

$$
T_{2 \kappa}=T_{2 \kappa}^{[1]}+T_{2 \kappa}^{[2]}+T_{2 \kappa}^{[3]},
$$

where

$$
\begin{aligned}
& T_{2 \kappa}^{[1]}=\frac{1}{N_{R}} \operatorname{Re}\left\{-2 M_{0}^{(0)}\left(\frac{1}{2} \frac{1}{2}\right) * M_{\kappa}^{(2)}\left(\frac{3}{2} \frac{1}{2}\right)\right\}, \\
& T_{2 \kappa}^{[2]}=\frac{1}{N_{R}} \operatorname{Re}\left\{\sqrt{2} M_{0}^{(0)}\left(\frac{3}{2} \frac{3}{2}\right) * M_{\kappa}^{(2)}\left(\frac{1}{2} \frac{3}{2}\right)\right\}, \\
& T_{2 \kappa}^{[3]}=\frac{1}{N_{R}} \operatorname{Re}\left\{\sqrt{2} M_{0}^{(0)}\left(\frac{3}{2} \frac{3}{2}\right)^{*} M_{\kappa}^{(2)}\left(\frac{3}{2} \frac{3}{2}\right)\right\},
\end{aligned}
$$

with

$$
N_{R}=\operatorname{Tr}\left(\boldsymbol{M} \boldsymbol{M}^{\dagger}\right)=6 \frac{d \sigma}{d \Omega} .
$$

Now our attention will be focused on the special scattering angle $\theta=90^{\circ}$ for simple considerations. Each component and the sum in Eq. (7) at $\theta=90^{\circ}$ are displayed for $T_{21}\left(T_{22}\right)$ in Fig. 6(a) [Fig. 6(b)] as functions of $E_{p}$. Figure 6(a) shows that the unfavorable effect of the BR-3NF on $T_{21}$ seen in Fig. 4 appears for $E_{p}>2 \mathrm{MeV}$, and the largest contribution arises from $T_{21}^{[3]}$ of the spin-quartet scattering. Since the quartet scalar amplitude $M_{0}^{(0)}\left(\frac{3}{2} \frac{3}{2}\right)$ is hardly affected by the 3 NFs as shown in Ref. [9], the spin-quartet tensor amplitude $M_{1}^{(2)}\left(\frac{3}{2} \frac{3}{2}\right)$ included in $T_{21}^{[3]}$ should be responsible for the effect. Adopting the Madison convention for the coordinate system, $\hat{z} \| \boldsymbol{k}_{i}$ and $\hat{y} \| \boldsymbol{k}_{i} \times \boldsymbol{k}_{f}$, one can write down $\left[C_{\ell_{i}}\left(\hat{k}_{i}\right)\right.$ $\left.\otimes C_{\ell_{f}}\left(\hat{k}_{f}\right)\right]_{1}^{(2)}$ as functions of $\cos \theta$ and $\sin \theta$. Referring to Eq. (5), we can restrict the invariant amplitude effective for $M_{1}^{(2)}\left(s_{i} s_{f}\right)$ to $\ell_{i}=\ell_{f}=1$ ( $P$ wave) as long as we are concerned with $\theta=90^{\circ}$, since the amplitudes of $\left(\ell_{i}, \ell_{f}\right)=(0,2)$ vanish because of the factor $\cos \theta \sin \theta$, and those of $\left(\ell_{i}, \ell_{f}\right)=(2,0)$ do not appear. In Eq. $(5), \ell_{i}\left(\ell_{f}\right)$ gives the orbital angular momentum in the initial (final) channel, when the $\theta$ dependence of $F^{(K)}\left(s_{i} s_{f} \ell_{i}\right)$ is neglected because of low-energy scattering. Then we conclude that the $P$-wave scattering in the spin-quartet state has a key to the unfavorable $3 \mathrm{NF}$ contribution to $T_{21}$.

The $3 \mathrm{NF}$ contribution to $T_{22}$ will be analyzed in a similar way. As seen in Fig. 6(b), $T_{22}^{[1]}$ gives small contributions to $T_{22}$. The other two terms $T_{22}^{[2]}$ and $T_{22}^{[3]}$ are accompanied by the scalar amplitude $M_{0}^{(0)}\left(\frac{3}{2} \frac{3}{2}\right)$ as Eqs. (9) and (10), which receives effectively no $3 \mathrm{NF}$ contribution as discussed above. The related tensor amplitudes $M_{2}^{(2)}\left(\frac{1}{2} \frac{3}{2}\right)$ and $M_{2}^{(2)}\left(\frac{3}{2} \frac{3}{2}\right)$ are also scarcely affected by the $3 \mathrm{NF}$. In fact, $M_{2}^{(2)}\left(\frac{3}{2} \frac{3}{2}\right)$ has been shown to be unaffected by the $3 \mathrm{NF}$ in neutron-deuteron scattering at $E_{n}=3 \mathrm{MeV}$ [9]. However, $N_{R}$ in the denominator is affected by the $3 \mathrm{NF}$, and most of the $3 \mathrm{NF}$ contributions to $T_{22}$ are produced by this effect. Since $N_{R}$ is proportional to 
TABLE I. $\chi^{2} /$ data of the AV18, AV18-GS, AV18-BR, and AV18-BR-SO $p d$ observables compared with the experimental data from Refs. [10-13].

\begin{tabular}{|c|c|c|c|c|c|c|}
\hline & $\sigma$ & $A_{y}$ & $i T_{11}$ & $T_{20}$ & $T_{21}$ & $T_{22}$ \\
\hline \multicolumn{7}{|l|}{$E_{p}=0.65 \mathrm{MeV}$} \\
\hline AV18 & 21 & 16 & 13 & 2.9 & 3.4 & 2.7 \\
\hline AV18-GS & 1.7 & 12 & 7.8 & 2.3 & 2.7 & 2.0 \\
\hline AV18-BR & 2.0 & 12 & 9.5 & 2.6 & 3.1 & 2.1 \\
\hline AV18-BR-SO & 1.7 & 2.4 & 1.5 & 2.6 & 3.2 & 2.1 \\
\hline \multicolumn{7}{|l|}{$E_{p}=1.0 \mathrm{MeV}$} \\
\hline AV18 & 51 & 164 & 60 & 3.7 & 3.8 & 4.2 \\
\hline AV18-GS & 2.8 & 115 & 28 & 1.4 & 2.8 & 1.5 \\
\hline AV18-BR & 2.8 & 116 & 37 & 1.6 & 3.4 & 1.7 \\
\hline AV18-BR-SO & 2.5 & 20 & 2.1 & 1.8 & 3.5 & 1.8 \\
\hline \multicolumn{7}{|l|}{$E_{p}=2.5 \mathrm{MeV}$} \\
\hline AV18 & 23 & 189 & 90 & 1.5 & 5.2 & 10 \\
\hline AV18-GS & 1.9 & 111 & 33 & 3.7 & 5.0 & 2.6 \\
\hline AV18-BR & 1.8 & 111 & 53 & 6.3 & 10 & 2.8 \\
\hline AV18-BR-SO & 2.2 & 1.9 & 10 & 5.5 & 8.9 & 3.1 \\
\hline \multicolumn{7}{|l|}{$E_{p}=3.0 \mathrm{MeV}$} \\
\hline AV18 & 25 & 160 & 114 & 3.2 & 7.1 & 18.0 \\
\hline AV18-GS & 2.3 & 95 & 47 & 5.1 & 8.3 & 3.6 \\
\hline AV18-BR & 2.2 & 94 & 74 & 10 & 20 & 4.3 \\
\hline AV18-BR-SO & 2.6 & 1.9 & 6.0 & 9.0 & 18 & 5.3 \\
\hline
\end{tabular}
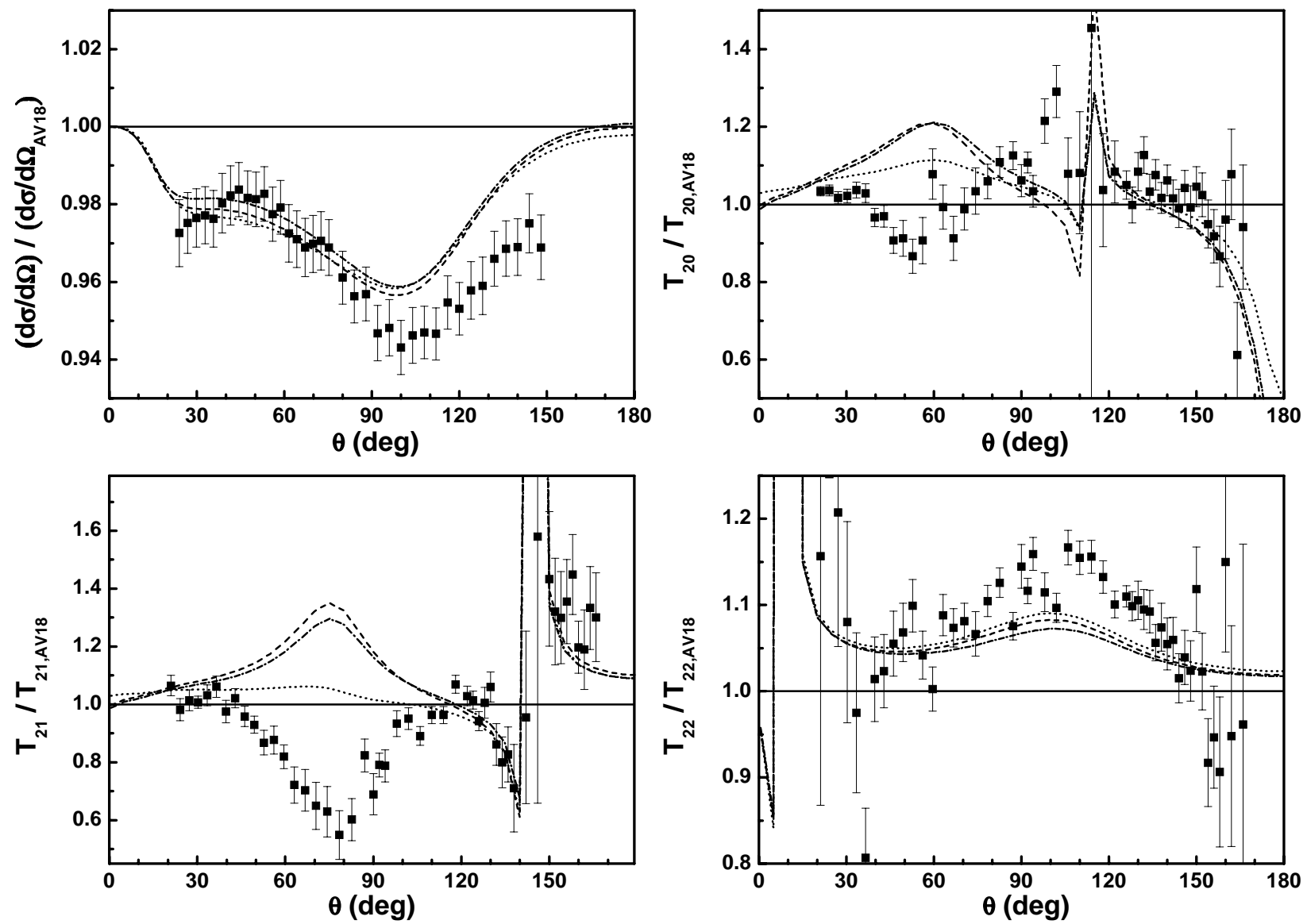

FIG. 4. Differential cross sections $d \sigma / d \Omega$ and tensor analyzing powers $T_{20}, T_{21}$, and $T_{22}$ at $E_{p}=3.0 \mathrm{MeV}$ divided by the theoretical values with the AV18. See the caption of Fig. 1 for the definitions of the theoretical curves. 


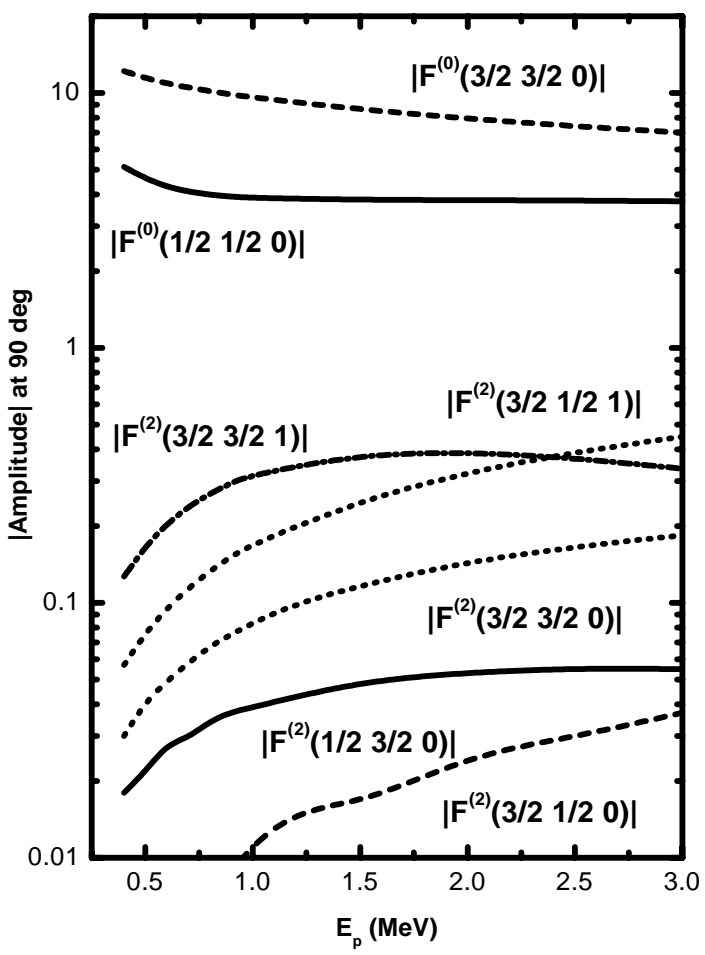

FIG. 5. Magnitudes of the invariant amplitudes $F^{(0)}\left(\frac{3}{2} \frac{3}{2} 0\right)$, $F^{(0)}\left(\frac{1}{2} \frac{1}{2} 0\right), F^{(2)}\left(\frac{3}{2} \frac{3}{2} 0\right), F^{(2)}\left(\frac{3}{2} \frac{1}{2} 0\right), F^{(2)}\left(\frac{1}{2} \frac{3}{2} 0\right), F^{(2)}\left(\frac{3}{2} \frac{3}{2} 1\right)$, and $F^{(2)}\left(\frac{3}{2} \frac{1}{2} 1\right)$ at $\theta=90^{\circ}$ as functions of $E_{p}$ calculated with the AV18.

the cross section, the successful improvements by the $3 \mathrm{NF}$ in $d \sigma / d \Omega$ and $T_{22}$ are achieved due to the same origin, the central component of the $3 \mathrm{NF}$.

We will conclude that at $E_{p}=3.0 \mathrm{MeV}$, the central part of the BR-3NF produces the successful contribution on the differential cross section as well as on the $T_{22}$ analyzing power,
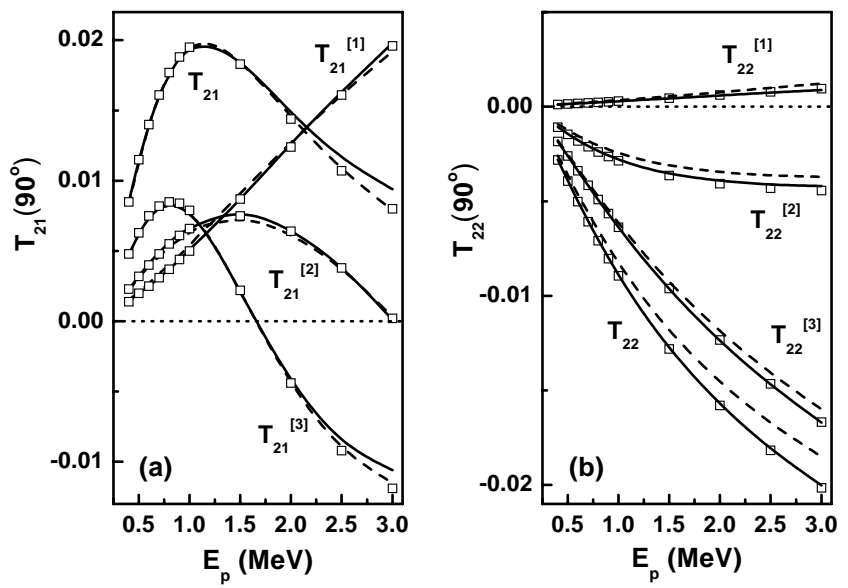

FIG. 6. Contributions of $T_{2 \kappa}^{(1)}\left(90^{\circ}\right), T_{2 \kappa}^{(2)}\left(90^{\circ}\right)$, and $T_{2 \kappa}^{(3)}\left(90^{\circ}\right)$ to $T_{2 \kappa}\left(90^{\circ}\right)$ as functions of $E_{p}$ for $\kappa=1$ (a) and $\kappa=2$ (b). The dashed lines, the solid ones, and the square blocks represent the calculations by the AV18, the AV18-BR, and the AV18-GS, respectively.

while the tensor part of the interaction gives the undesirable contribution to $T_{21}$. The $3 \mathrm{NF}$ tensor effect on $T_{21}$ is not observed below $1 \mathrm{MeV}$ as seen in Table I, and Fig. 6 suggests that it may become appreciable above $2 \mathrm{MeV}$. Thus, precise measurements of the observables for the $p d$ scattering are highly desirable in such an energy region for the study of the $3 \mathrm{NF}$ tensor effect. Also it will be interesting to examine the energy dependence of the effect up to higher energies, where several problems of the tensor analyzing powers have been reported $[16,17]$.

This research was supported by the Japan Society for the Promotion of Science, under a Grant-in-Aid for Scientific Research (Grant No. 13640300). The numerical calculations were supported, in part, by the Computational Science Research Center, Hosei University, under Project No. lab0003.
[1] T. Sasakawa and S. Ishikawa, Few-Body Syst. 1, 3 (1986).

[2] S. Ishikawa, Few-Body Syst. (to be published), nucl-th/0206064.

[3] J. L. Friar and G. L. Payne, in Coulomb Interactions in Nuclear and Atomic Few-Body Collisions, edited by F. S. Levin and D. A. Micha (Plenum, New York, 1996), p. 97.

[4] A. Kievsky, J.L. Friar, G.L. Payne, S. Rosati, and M. Viviani, Phys. Rev. C 63, 064004 (2001).

[5] A. Kievsky, S. Rosati, W. Tornow, and M. Viviani, Nucl. Phys. A607, 402 (1996).

[6] R.B. Wiringa, V.G.J. Stoks, and R. Schiavilla, Phys. Rev. C 51, 38 (1995).

[7] H.T. Coelho, T.K. Das, and M.R. Robilotta, Phys. Rev. C 28, 1812 (1983).

[8] A. Kievsky, Phys. Rev. C 60, 034001 (1999).

[9] S. Ishikawa, M. Tanifuji, and Y. Iseri, Phys. Rev. C 66, 044005 (2002).

[10] C.R. Brune, W.H. Geist, H.J. Karwowski, E.J. Ludwig, K.D. Veal, M.H. Wood, A. Kievsky, S. Rosati, and M. Viviani, Phys.
Rev. C 63, 044013 (2001).

[11] K. Sagara, H. Oguri, S. Shimizu, K. Maeda, H. Nakamura, T. Nakashima, and S. Morinobu, Phys. Rev. C 50, 576 (1994).

[12] S. Shimizu, K. Sagara, H. Nakamura, K. Maeda, T. Miwa, N. Nishimori, S. Ueno, T. Nakashima, and S. Morinobu, Phys. Rev. C 52, 1193 (1995).

[13] M.H. Wood, C.R. Brune, B.M. Fisher, H.J. Karwowski, D.S. Leonard, E.J. Ludwig, A. Kievsky, S. Rosati, and M. Viviani, Phys. Rev. C 65, 034002 (2002).

[14] S. Ishikawa, Phys. Rev. C 59, R1247 (1999).

[15] M. Tanifuji and K. Yazaki, Prog. Theor. Phys. 40, 1023 (1968).

[16] A. Kievsky, M. Viviani, and S. Rosati, Phys. Rev. C 64, 024002 (2002).

[17] K. Sekiguchi, H. Sakai, H. Witała, W. Glöckle, J. Golak, M. Hatano, H. Kamada, H. Kato, Y. Maeda, J. Nishikawa, A. Nogga, T. Ohnishi, H. Okamura, N. Sakamoto, S. Sakoda, Y. Satou, K. Suda, A. Tamii, T. Uesaka, T. Wakasa, and K. Yako, Phys. Rev. C 65, 034003 (2002). 